\title{
Conventional bitewing radiography
}

\author{
Falk Schwendicke ${ }^{1} \cdot$ Gerd Göstemeyer $^{2}$ (D)
}

Received: 6 August 2020 / Accepted: 9 September 2020 / Published online: 22 October 2020

(c) The Author(s) 2020

\begin{abstract}
Accurate detection and diagnosis of carious lesions is the prerequisite for application of the most effective caries management approach. However, the accuracy of proximal caries detection using visual-tactile assessment is limited. Additional diagnostic measures should therefore be performed to increase the validity of the diagnosis. Amongst various caries detection aids available, bitewing radiographs remain the gold standard for detection of proximal caries lesions. Here we describe a method for caries diagnosis using standardized bitewing holders. With this method the bitewing holders can be placed more precisely and reproducibly in the oral cavity. This approach increases accuracy of bitewing radiographs, which, due to the reproducible placement of the x-ray film, allows for monitoring of carious lesions long term.
\end{abstract}

Keywords Bitewing $\cdot$ Caries detection $\cdot$ Caries diagnosis · Radiography

\section{Quick reference/description}

Detection of carious lesions, especially on non-accessible (proximal) surfaces, is difficult through visual and tactile examination. Early detection of non-cavitated proximal carious lesions can be crucial, e.g., for non- or micro-invasive interventions. Bitewing radiography is an efficient imaging method that can be employed along with visual and tactile examination to increase the accuracy of the caries diagnosis.

\section{Indications}

- Early detection of primary coronal carious lesions on occlusal and proximal surfaces.

Gerd Göstemeyer

Gerd.goestemeyer@charite.de

1 Department of Oral Diagnostics, Digital Health and Health Services Research, CharitéUniversity, Berlin, Germany

2 Department of Operative and Preventive Dentistry, Charité-University, Berlin, Germany 
- Detection of proximal root caries.

- Assessment of the quality of restorations and detection of secondary caries in the proximal area.

- Detection of lesion extension in enamel and dentinal carious lesions.

- Long-term monitoring of carious lesions.

\section{Materials/instruments}

- Standardized or customizable bitewing holders (e.g., Icon X-ray Holder, DMG, Hamburg).

- For customizable bitewing holders wax or hard-curing silicone.

- Cow horn-ended instrument.

- Orthodontic rings.

\section{Procedure}

Early active interproximal carious lesions can be managed non- or micro-invasively, i.e., without the need of placing a restoration. However, once a lesion has reached a certain depth, loss of surface integrity is likely and the lesion requires invasive restorative interventions that are irreversible and with a limited longevity. Invasive restorations predispose the tooth to repeated and expensive re-interventions and result in substantial destruction of sound dental tissues to gain access to the lesion. Therefore, if indicated, non-invasive or micro-invasive methods are preferable to restorative measures.

The main objectives of current caries detection methods are:

- Accurate detection and grading according to extension and activity of the lesion.

- To provide appropriate information about surface status (i.e., cavitated or noncavitated).

- Allow for reproducible monitoring of caries lesions long term.

On the basis of these requirements, an ideal caries detection method should aid visual and tactile examination by providing information about the presence, depth and surface status of a lesion. Bitewing radiography is one such adjunctive imaging method that assists the early detection of proximal carious lesions.

Bitewing radiography has a low sensitivity for the detection of early proximal lesions that extend only into enamel. This leads to the assumption that very early lesions (extending in the outer enamel histologically) will usually remain undetected. The sensitivity of detection increases with more advanced dentinal or cavitated lesions. The specificity of bitewing radiography is high (around or above 0.9), suggesting that only around $10 \%$ of the detected lesions are actually sound surfaces (Table 1).

As bitewing radiography is usually used for long-term patient monitoring, it is performed at repeat intervals (every 12-24 months). Hence, the risk of underdetection reduces with each subsequent examination. Nowadays, as most carious 
lesions progress gradually, the sensitivity and specificity of bitewing radiography are apt for early detection of lesions without the enhanced risk of false positive detections. The accuracy of bitewing radiography to detect proximal root caries is comparable to that of proximal coronal lesions. It is vital that the cervical beamout artefact often seen radiographically on root surfaces near the alveolar ridge is wrongly diagnosed.

The proximal carious lesions identified on radiographs can be scored according to their extent. A wide array of scoring systems is available for detecting the extent of proximal lesions. One system is based on dividing the proximal area of the tooth into four parts: two (outer and inner) halves in enamel and two (outer and inner) halves in dentin (termed as D1-D4 for decayed or R1-4 for radiographically decayed 1-4). A more accurate system divides the enamel into an outer half and an inner half (E1, E2) and the dentin into an outer third, a middle third and an inner third (D1-3) (Fig. 1).

This system provides adequate description of the lesions and aids in making treatment decisions. This scoring system can also monitor carious lesions over time, and thus confirm whether a lesion actually shows clinical activity (along with clinical lesion activity determination). Depending on lesion depth, the lesions can be managed differently (Table 2).

Bitewing radiography rarely permits the detection of surface cavitation, but it can determine the level from which cavitation is likely to occur. Studies suggest that about half of proximal carious lesions extending into the outer half of dentin are cavitated. However, the probability of occurrence of surface cavitation varies widely: only a third of the carious lesions radiographically extending into dentin are observed to be cavitated in low-risk patients, while almost all carious lesions extending into dentin are seen to be cavitated in patients with high caries risk. However, this information is based on data from older studies and more recent lesion progression rates seem to be slower. Hence, it can be speculated that the probability of cavitation of lesions extending to outer dentin has reduced.

As to the difficulty in determining the surface status of non-accessible proximal carious lesions, clinicians should make a conscious effort to clinically confirm the radiographic findings once a presumably cavitated lesion was detected. In case of doubtful lesions, non-restorative treatment modalities including non- or microinvasive therapy can be implemented. Such lesions can always be restored at a later

Table 1 Advantages and disadvantages of bitewing radiography

\begin{tabular}{ll}
\hline Advantages & Disadvantages \\
\hline $\begin{array}{l}\text { Adequate sensitivity, also for early } \\
\text { proximal lesions }\end{array}$ & Uncertain determination of surface status \\
$\begin{array}{l}\text { Can be used and applied in a } \\
\text { clinical setting and is useful for } \\
\text { research }\end{array}$ & Low sensitivity if lesions are not located within the beam \\
$\begin{array}{l}\text { Facilitates long-term monitoring of } \\
\text { lesions if used with standardized } \\
\text { or individualized holders }\end{array}$ & Exposure to ionizing radiation \\
& Requires ion source and equipment \\
\hline
\end{tabular}




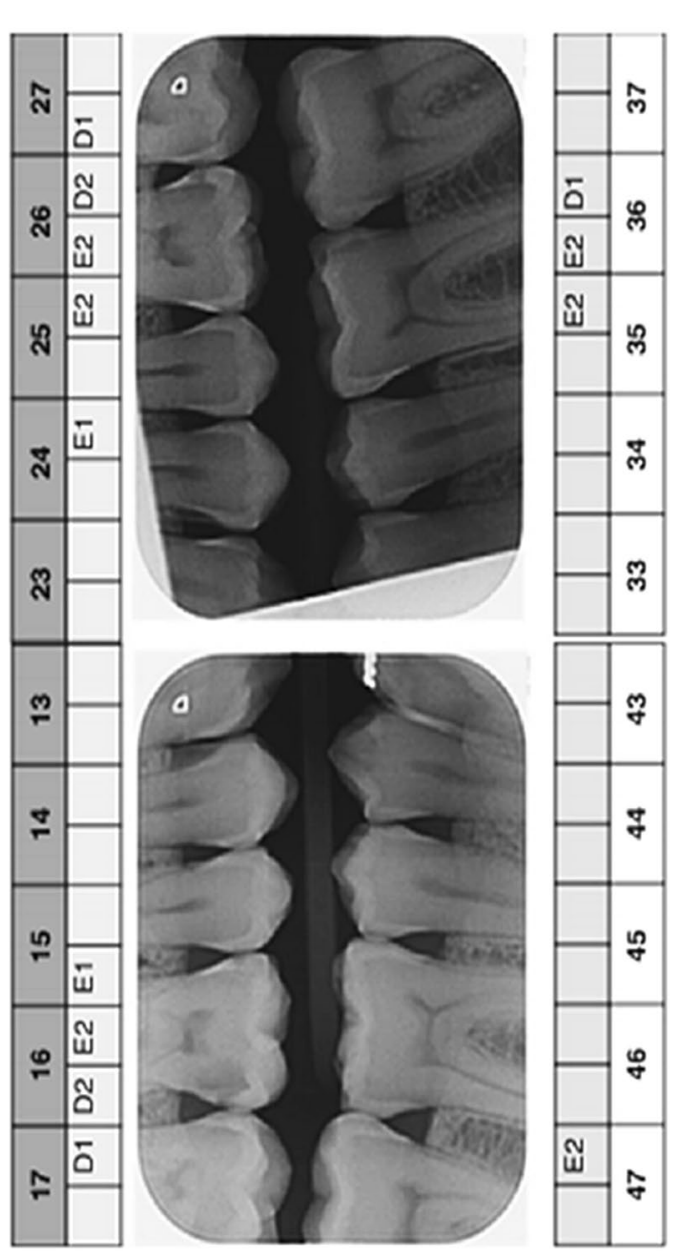

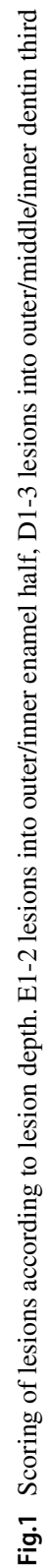


Table 2 Management of carious lesions depending on scoring status

\begin{tabular}{ll}
\hline Scored carious lesions & Management \\
\hline E1-D1 lesions & Non-restorative management \\
D2-D3 lesions & $\begin{array}{c}\text { Restorative management depending on } \\
\text { their proximity to pulp }\end{array}$ \\
\hline
\end{tabular}

time if required. Several methods can be used to double-check the surface status of doubtful lesions. A cow horn-ended instrument can be used for gentle probing without any force as forceful probing can cause cavitation of demineralized, but surface-intact enamel. In addition, orthodontic rings can be used for the separation of suspected proximal spaces to facilitate direct inspection.

Radiographs are less suitable for the detection of carious lesions on other surfaces than in the proximal area. The sensitivity of radiographs to detect occlusal lesions is similar to that of proximal lesions, while its specificity is lower. The extent of occlusal lesions is usually underestimated on radiographs. Lesions that radiographically appear to 'just' extend into the dentin are often deeper clinically (Fig. 2).

Bitewing radiography is also a valuable aid for the detection of secondary caries. However, as with primary carious lesions, it is also usually more suitable for lesions at proximal restoration margins, where the lesion is situated 'within' the X-ray beam. The sensitivity and specificity of bitewing radiography for the detection of secondary caries lesions is similar to that of other tools such as laser-fluorescence.

As ionizing radiation is a major pitfall of bitewing radiography, it restricts its repeatability and application in young children. To reduce radiation exposure, clinicians should strictly follow the rules of radiation protection (keeping radiation dose as low as reasonably possible). They should also limit their recommendation of bitewing radiography in children. The time intervals for obtaining bitewing radiographs should be planned and adjusted according to the caries risk.

\section{Clinical application}

The following considerations are recommended when prescribing, obtaining and interpreting bitewing radiographs:

- Use an optimal X-ray projection that aims to be directed straight at the proximal space, i.e., parallel to the oro-buccal tooth axis.

- Bitewing radiographs should be standardized or used alongside with individualized bitewing holders. To customize and individualize bitewing holders, the patients are asked to bite into small pieces of wax or hard-curing silicone (fixation mass) placed onto the holder. This fixation mass facilitates placement of the bitewing holder in the exact same position with repeated bitewings and may aid to ensure the optimal X-ray projection (see above) for the radiograph. Thus, allowing valid comparison and monitoring of lesions over time. Note that indi- 


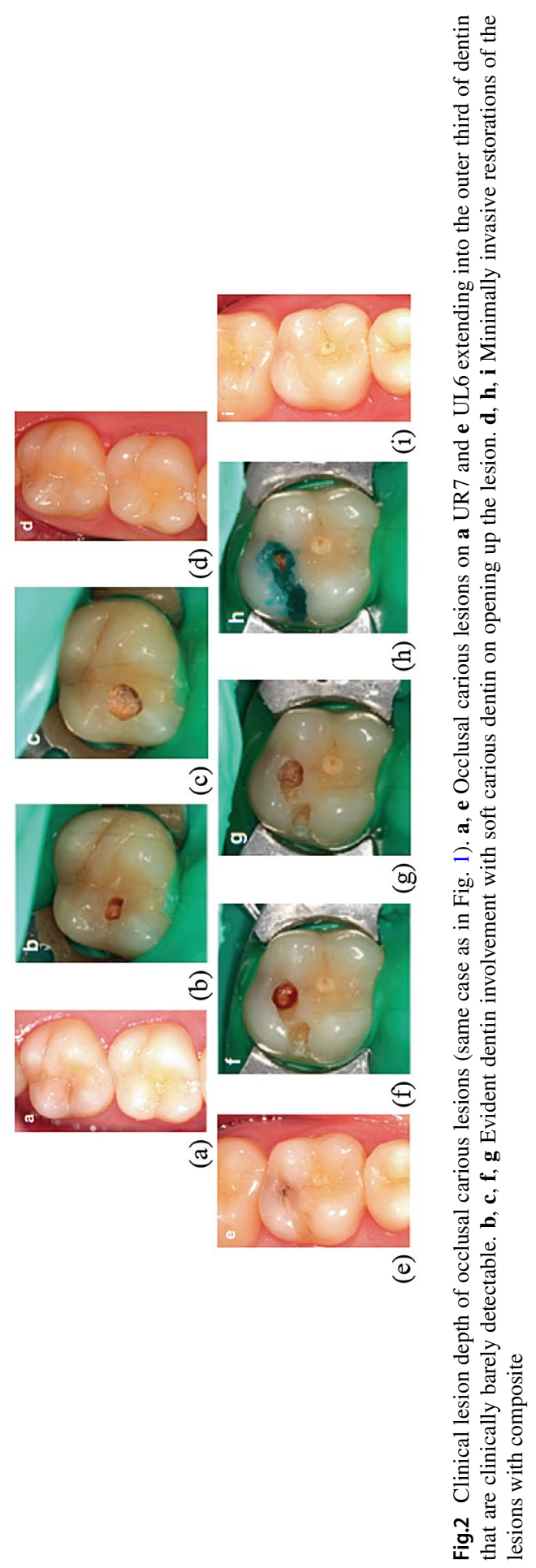


(a)

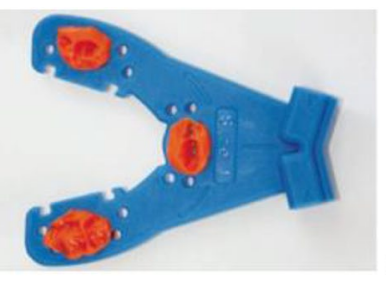

(b)

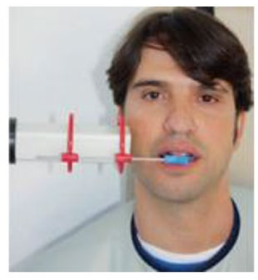

Fig.3 a Individualized bitewing holders. b Individualized holders allowing consistent positioning of the $\mathrm{X}$-ray cone and film for monitoring of carious lesions over time

vidualized bitewing holders are helpful only in adults with permanent dentition without orthodontic movement of teeth (Fig. 3).

- The radiographic findings should be recorded on an appropriate scale to increase the accuracy of the diagnosis - an accurate diagnosis scale facilitates appropriate treatment decisions.

- In case of uncertainty based on bitewing radiographs, cavitation status of carious lesions should be determined and confirmed clinically via gentle probing or the use of orthodontic rings for tooth separation.

- The uncertainty of any diagnosis should be integrated into the decision-making of treatment interventions. Non-restorative treatment modalities should be selected and implemented, whenever in doubt.

\section{Pitfalls and complications}

- Bitewing radiography is not helpful for detecting caries on smooth surfaces (buccal and lingual).

- Individualized bitewing holders are not useful in children due to continued growth and tooth exfoliation or during orthodontic treatment due to tooth movement.

- Changes in X-ray projection can cause some carious lesions to falsely appear as 'progressing' or 'regressing'.

- Ionizing radiation is a major pitfall of bitewing radiography and limits its repeatability and application in young children.

- Bitewing radiography poses a risk of under-detection for early proximal lesions.

- Bitewing radiography carries an increased risk of underestimation of lesion extension in occlusal lesions.

Funding Open Access funding enabled and organized by Projekt DEAL.

Open Access This article is licensed under a Creative Commons Attribution 4.0 International License, which permits use, sharing, adaptation, distribution and reproduction in any medium or format, as long as you give appropriate credit to the original author(s) and the source, provide a link to the Creative Commons licence, and indicate if changes were made. The images or other third party material in this article 
are included in the article's Creative Commons licence, unless indicated otherwise in a credit line to the material. If material is not included in the article's Creative Commons licence and your intended use is not permitted by statutory regulation or exceeds the permitted use, you will need to obtain permission directly from the copyright holder. To view a copy of this licence, visit http://creativecommons.org/licen ses/by/4.0/.

\section{Further reading}

1. Ferreira Zandona A, Longbottom C (eds) (2019) Conventional bitewing radiographs detection and assessment of dental caries. Springer, Berlin. https://doi.org/10.1007/978-3-030-16967-1_11

2. Schwendicke F, Tzschoppe M, Paris S (2015) Radiographic caries detection: a systematic review and meta-analysis. J Dent 43:924-933

3. Innes N, Schwendicke F (2017) Dentists' thresholds for restorative intervention in carious lesions: systematic review and meta-analysis. J Dent Res 96(5):501-508

4. Wenzel A (2014) Radiographic display of carious lesions and cavitation in approximal surfaces: advantages and drawbacks of conventional and advanced modalities. Acta Odontol Scand 72:251-264

5. Brouwer F, Askar H, Paris S, Schwendicke F (2016) Detecting secondary caries lesions: a systematic review and meta-analysis. J Dent Res 95:143-151

Publisher's Note Springer Nature remains neutral with regard to jurisdictional claims in published maps and institutional affiliations. 